\title{
HIGH COMPLIANT SERIES ELASTIC ACTUATION FOR THE ROBOTIC LEG SCARLETH
}

\author{
MARCO HUTTER, C. DAVID REMY, MARK A. HOEPFLINGER, \\ ROLAND SIEGWART ${ }^{*}$ \\ Autonomous Systems Lab, Institute of Robotics and Intelligent Systems, Swiss Federal \\ Institute of Technology (ETHZ),Zurich, Switzerland, mahutter@ethz.ch
}

\begin{abstract}
This paper presents the actuation system of the robotic leg ScarlETH. It was developed specifically for a quadrupedal robot and is designed to achieve fast position control as well as accurate joint torque control. It introduces strong passive dynamics to create an efficient running behavior. High spring compliance with low damping in combination with a cascaded, motor velocity based, control structure was successfully tested in simulation and experiments. Final tests with the entire leg demonstrate that the system can perform a hopping motion providing only positive actuator power.
\end{abstract}

\section{Introduction}

Series elastic actuation (SEA) is a favored actuation approach when it comes to dynamic maneuvers in legged locomotion [1]. The elastic decoupling of the actuator from the joint is not only beneficial due to its mechanical low-pass filter characteristic that inherently increases safety for the environment [2] and that additionally protects the actuator from peak loads at landing impact [3]. Using the spring as a force element also allows performing torque control by controlling the deflection of the spring. The principle "the stiffer, the better" dominated classical robotic applications for a long time, and a similar tendency can be observed in the field of series elastic actuation: Such actuators are usually evaluated regarding force control bandwidth, which makes stiff springs clearly preferable due to the smaller motor travel distances that are necessary. In contradiction thereto, we would like to make the point that a great benefit of high compliance in combination with low damping is that it allows storing and releasing a substantial amount of energy during ground contact phases. These principles are adapted from nature, where tendons and elastic elements in muscles largely contribute to the bouncing behavior of dynamic maneuvers such as running. Provided that the stiffness is well chosen, this approach significantly

\footnotetext{
* This research was supported by the Swiss National Science Foundation through the National Centre of Competence in Research Robotics and the Hans-Eggenberger-Foundation.
} 
increases the energy efficiency and reduces the power consumption of the actuators - a crucial element when building legged systems for highly dynamic maneuvers.

In the present paper, we will introduce the series elastic actuation developed for ScarlETH (Series compliant articulated robotic leg), a single leg prototype for a quadruped running robot. The hardware as well as the controller is designed to achieve accurate torque control with a good reference action as well as good disturbance rejection and fast position control including active damping. In addition, with properly tuned joint stiffness, this setup allows to optimally exploiting the natural dynamics of the system such that efficient running is possible.

a) hip flextion

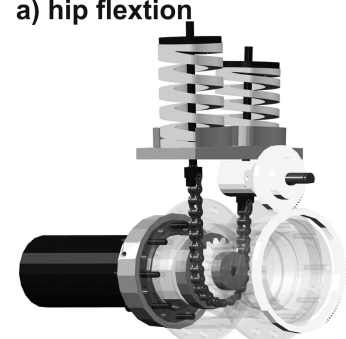

b) hip abduction

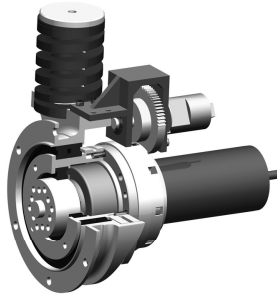

c) knee flexion

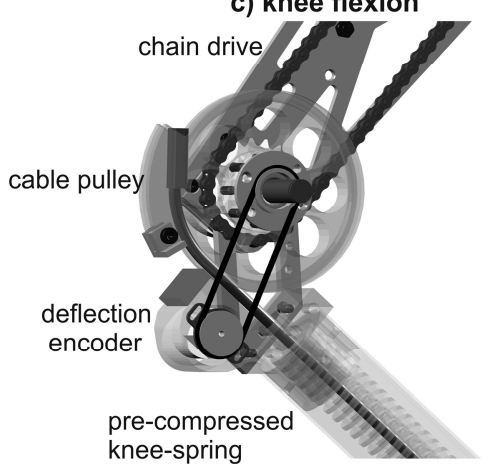

d) ScarlETH

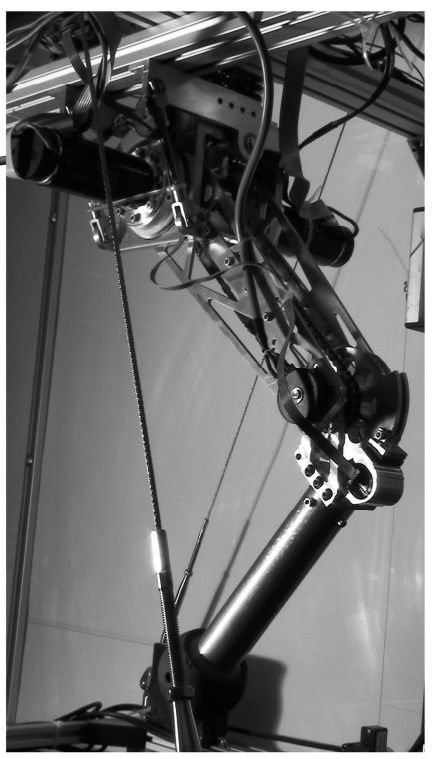

Figure 1 ScarlETH (d) is driven by 3 high compliant series elastic actuators for hip flexion (a), hip abduction (b), and knee flexion (c). A chain/cable pulley design in combination with compression springs in all joints allows placing all motors at the hip joint such that the segments are lightweight (beneficial for fast motion, impact loss, and CoG close to the hip joint) and that a huge range of motion is achieved (complete knee retraction $\varphi_{\text {knee }}^{\max }=170^{\circ}$ and hip flexion $\left.\varphi_{h i p}^{\max }= \pm 85^{\circ}\right)$. All joints are actuated by 200 watt EC-motors in combination with harmonic drive units. Accurate force control is possible due to high linearity in the spring characteristics and very accurate deflection ( $\left.80^{\prime} 000 \mathrm{qc} / \mathrm{rev}\right)$ and motor position (160’000 qc/rev) encoding. 


\section{System description}

The fully actuated leg (Fig. 1) consists of three series elastic actuators for knee and hip flexion/extension as well as hip adduction/abduction. Powerful maxon motors (RE35 90W, EC4pole30 200W ${ }^{\dagger}$ ) in combination with harmonic drive units (CSG14, 1:100 reduction ${ }^{*}$ ) are connected over a chain and a steel cable pulley design with linear compression springs. This allows placing all actuators directly at the hip joint (Fig. 1) and facilitates not only a huge range of motion but also minimizes the inertia of the thigh and shank segment and hence the impact collision losses. The harmonic drive gearbox, the output shaft, as well as the springs are included in a highly integrated design that was optimized for weight (total leg weight of $4.5 \mathrm{~kg}$ ).

All springs are pre-loaded, resulting in an entirely backlash free design. A series of equal sized springs with different stiffness allow an optimal system tuning not only in simulation but also in experiments. In the present work, the knee stiffness is approximately $36 \mathrm{Nm} / \mathrm{rad}$ with a corresponding maximal joint deflection of $60^{\circ}$, the hip joint about $70 \mathrm{Nm} / \mathrm{rad}$ with a maximal deflection of $30^{\circ}$.

Each motor is controlled by a maxon EPOS controller that features current and velocity control. They are connected by a CAN bus to the low level FPGA controller on a compactRIO ${ }^{\S}$ system of National Instruments where joint position as well as torque controllers are implemented. The torque is measured using an incremental deflection encoder ${ }^{* *}$ with high resolution (80'000 qc/turn) connected to a sensor board.

\section{Control}

Controlling a legged robotic system generally poses two major, but inherently different control problems. On one side, during non-contact phases, accurate and fast position control is required for leg positioning. Due to the high joint compliance, this requires active damping: In ScarlETH, we solved this problem by an LQR controller design based on motor as well as joint position and speed measurements. A more detailed description can be found in [4], which is directly compatible with the presented control structure in this paper.

\footnotetext{
${ }^{\dagger}$ maxon motor AG, www.maxonmotor.ch

$\$$ Harmonic Drive AG, www.harmonicdrive.de

$\S$ compactRIO, National Instruments, www.ni.com/compactrio/

${ }^{* *}$ AEDA-3300, AVAGO Technologies, www.avagotech.com
} 


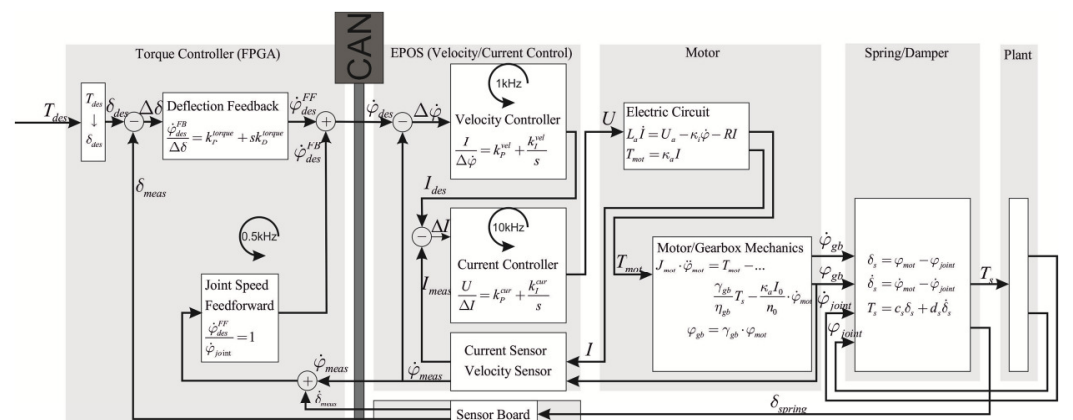

Figure 2 SEA controller structure: a cascaded structure with current control and velocity control on a maxon EPOS controller and a torque as well as a position controller on a NI FPGA. For high compliant springs with large deflections this velocity based torque control is beneficial.

The second control strategy, namely accurate joint torque control, is required during support phase. This facilitates virtual model control techniques [5], operational space control [6], or low gain walking control [7], which is clearly beneficial when dealing with uncertainties in the environment. In the standard SEA setup, a PID torque controller with a subordinate current controller is used [8]. In contrast thereto, some studies have demonstrated that a cascaded structure with additionally closing the motor velocity loop is beneficial [9],[10], especially to compensate undesired gearbox effects. In our application of the SEA, high compliance with low damping enforces large deflections and hence long motor shaft travel distances. Therefore, and because we have a setup with a high performance velocity controller at hand, we propose the cascaded control structure depicted in Fig. 2.

\subsection{Setup}

The linearized continuous time model of the entire actuation system is summarized in Fig. 2. System identification of the spring characteristic showed nearly perfect linearity with low damping and virtually no hysteresis in all joints [4], hence torque control remains equivalent to spring deflection control (damping is negligible). In this cascaded control structure the actual torque controller is implemented on the FPGA where it runs in a $500 \mathrm{~Hz}$ loop and creates the set points for the subordinate velocity and current controllers which are running with $1 \mathrm{kHz}$ and $10 \mathrm{kHz}$ respectively. The entire structure was rebuilt in simulation including saturation, discretization, and filtering effects. Detailed analysis of the individual loops in different model identification tests showed great equivalence to the simulation (Fig. 3). Both, current and velocity gains were tuned on a simplified test bench, resulting in a closed loop velocity bandwidth of about $100 \mathrm{~Hz}$ (b) and a step response time of about $5 \mathrm{~ms}$ (a). 
a) velocity step

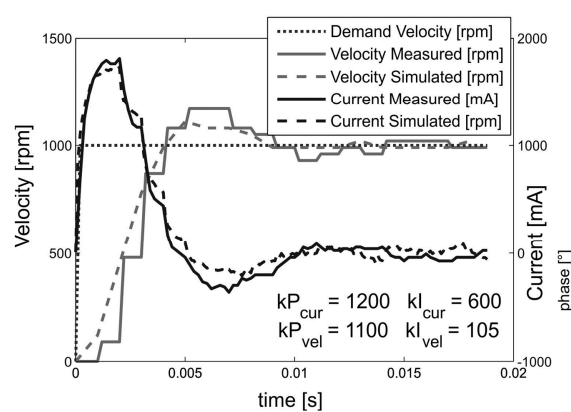

b) velocity frequency response

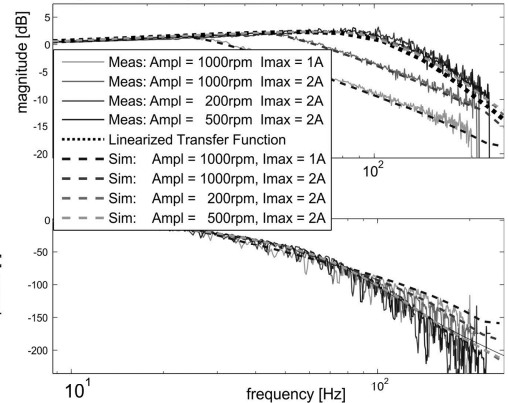

Figure 3 Experimental and simulated performance of the presented control structure depicting a nearly perfect matching of simulation and experiment (a) and a velocity control bandwidth of about $100 \mathrm{~Hz}(\mathrm{~b})$.

\subsection{Torque control design}

Based on detailed simulations as well as experiments, the torque controller was tuned with regard to two design criteria:

Firstly, fast reference signal-following must be achieved; i.e, a high closed loop torque bandwidth is required in combination with high robustness. Fig. $4 \mathrm{a}$ shows a step response time of about $30 \mathrm{~ms}$ for an input of $3 \mathrm{Nm}$. The closed loop transfer function (Fig. 4b) emphasizes the drawback of high compliance: with increasing amplitudes, the bandwidth substantially drops due to saturation effects. Nevertheless, the TF estimation with the entire model and the validated control parameters varies between $28 \mathrm{~Hz}$ for $1 \mathrm{Nm}, 15 \mathrm{~Hz}$ for $5 \mathrm{Nm}$ and $11 \mathrm{~Hz}$ for $10 \mathrm{Nm}$. The closed loop system is robust with a phase margin of more than $85^{\circ}$.

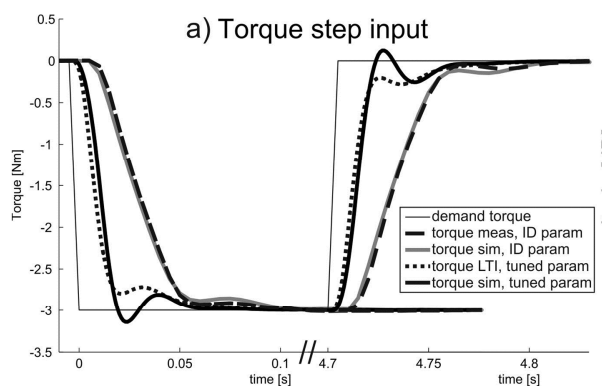

b) Transfer estimation

Figure 4 Experimental and simulated performance of the presented SEA control structure: the time response shown in (a) was used to validate controller implementation on the hardware (dashed and gray curve match well) and to tune the system for high performance. A bandwidth shown in (b) of about $28 \mathrm{~Hz}$ is achieved for small amplitudes; about $11 \mathrm{~Hz}$ remain when saturation effects occur. Keeping the integrator gains small results in a negligible overshoot $\left(T_{\max }<1 \mathrm{~dB}\right)$ and good robustness with a phase margin of more than $85^{\circ}$. 
Due to the high compliance, the resulting bandwidth is poor compared to systems with stiffer springs [11], still they are within the range of Pratt's electrical SEAs $(5-25 \mathrm{~Hz}$ [1]) and above the frequency range that can be found in human motion $(4-8 \mathrm{~Hz}[12])$.

Secondly, next to a fast reference signal response, a good disturbance rejection is crucial. This can be tested in a zero torque behavior with modulated external joint deflection. Using only a PI controller on the deflection leads to substantial torque ripples[9], mainly caused by a phase delay (dash-dotted line in Fig. 5a) between the joint and motor angles. As depicted in Fig. 5b, the sensitivity of the PI controller has a flat ascent requiring for higher integral gains to keep the torque ripple small. This would clearly influence the reference following behavior, having a negative influence on the robustness.
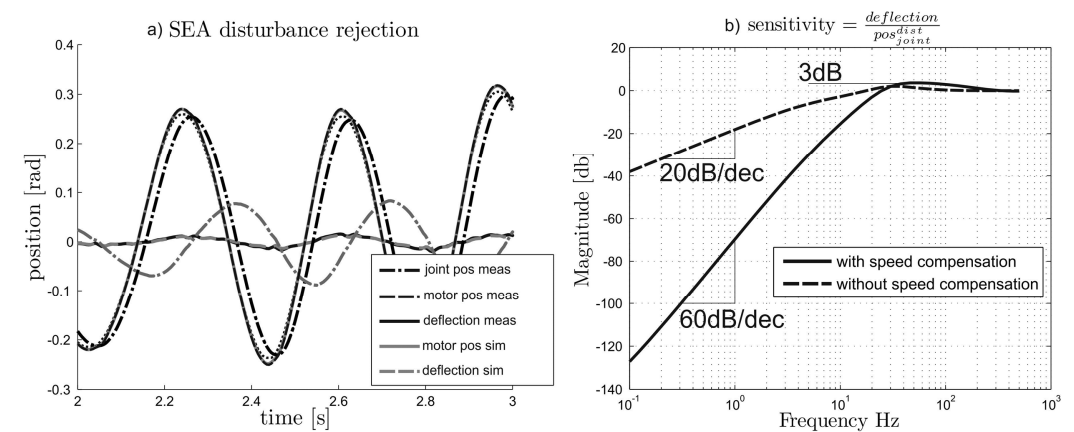

Figure 5 Significant improvement regarding disturbance rejection is achieved by disturbance compensation based on joint velocity measurement. Without that, high integral gains that also influence the closed loop reference signal behavior would be needed to achieve a good zero torque behavior.

Thanks to the backlash free mechanical design (pre-compressed springs and harmonic drive gearboxes) and the accurate measurements of motor position and spring deflection, we have very direct means of measuring the actual disturbance. Because of the high resolution (160'000 counts/rev at the motor, 80 '000 counts/rev for the deflection) encoders show minimal discretization noise and hence provide accurate joint speed estimation. Using these inputs, a disturbance compensator is included. This compensator has no influence on the properties of the closed loop controller, such that the previously achieved stability and robustness properties are conserved. Due to the low-pass behavior of the velocity controlled motor, the joint velocity input can be directly used as a feet forward path, inherently ensuring that the output value is independent of the input value for the stationary case since $\dot{\varphi}_{\text {mot }}(s=0)=\dot{\varphi}_{\text {joint }}(s=0)$. The sensitivity is still bounded by $3 \mathrm{~dB}$ but shows a much better behavior for lower 
frequencies (at which the system is usually operated) (Fig. 5b). The zero torque behavior was successfully tested in experiments. The measured joint disturbance was then used as simulation input and compared against the measurements. A significant improvement to the controller without disturbance compensation can be observed. At this point, the high compliance clearly helps, since the time constant for the required reaction is lower than in a system using stiff springs.

a) power

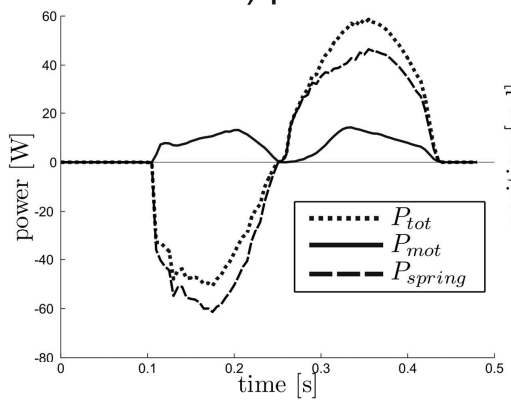

b) position

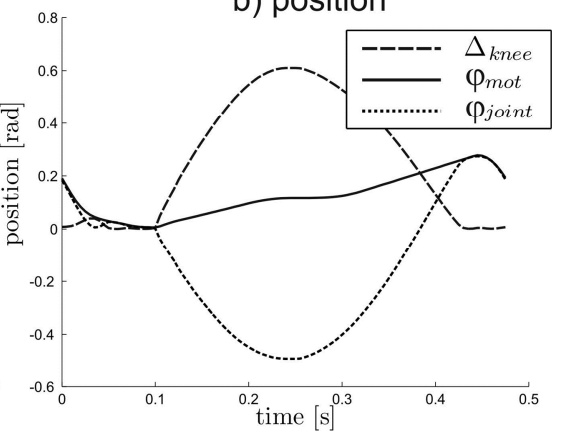

Figure 6 A modified Raibert style controller allows for efficient hopping with the presented hardware. During the whole stance phase, the knee motor is expanded (b) and provides exclusively positive power to the knee joint. When comparing the actuator power with the energy that is being stored in the spring (a) it becomes evident that the motion is dominantly driven by the natural dynamics. The motor only has to compensate for energetic losses and constitutes to less than $20 \%$ of the total joint work.

\section{Efficient running}

The main advantage of using high compliances with low damping becomes evident when it comes to perform highly dynamic maneuvers. In the present setup, knee joint deflections of $60^{\circ}$ allowed to store up to 30 Joules of energy. This corresponds to a vertical excursion of the total system's mass by $0.5 \mathrm{~m}$. A detailed description of the controller for 2D running can be found at [4]. During stance phase, we additionally ensured that the knee motor which is the main energy source delivers only positive power by an expansion speed $\dot{\varphi}_{\text {mot }}>0$. Fig. 6 shows the power distribution as well as the joint angles during a stance period in steady state $\left(\dot{x}_{H i p}=0\right)$ hopping. The motor itself provides exclusively positive power during the deceleration and acceleration phase. Approximately $20 \%$ of the total energy is introduced by the motor. The remaining fluctuations are created passively. 


\section{Conclusion and Outlook}

In this paper we presented the actuation system of the prototype leg ScarlETH that was built for a quadruped robot. This system combines all aspects needed for a versatile, fast, and efficient legged platform: high damped position control for precise leg positioning, accurate torque control during stance phase, and high compliant energy storage to generate efficient and fast running behaviors.

\section{References}

1. Pratt, J.E. and B.T. Krupp, Series elastic actuators for legged robots. Unmanned Ground Vehicle Technology Vi, 2004. 5422: p. 135-144, 618.

2. Wyeth, G. and G. Walker, Assessing the safety of a velocity sourced series elastic actuator using the head injury criterion, in Proceedings of Australasian Conference on Robotics and Automation 2007, M. Dunbabin and M. Srinivasan, Editors. 2007.

3. Pratt, G.A., et al., Stiffness Isn't Everything, in The 4th International Symposium on Experimental Robotics IV. 1997, Springer-Verlag.

4. Hutter, M., et al., ScarlETH: Design and Control of a Planar Running Robot, in in prep. for IROS 2011. 2011: San Francisco, USA.

5. Pratt, J., et al., Virtual model control: An intuitive approach for bipedal locomotion. International Journal of Robotics Research, 2001. 20(2): p. 129143.

6. Sentis, L., Compliant Control of Whole-Body Multi-Contact Behaviors in Humanoid Robots, in Motion Planning for Humanoid Robots. 2009, Springer Global Editorial.

7. Kalakrishnan, M., et al. Fast, Robust Quadruped Locomotion over Challenging Terrain. in ICRA 2010. 2010. Anchorage, AK.

8. Robinson, D.W., et al. Series elastic actuator development for a biomimetic walking robot. in International Conference on Advanced Intelligent Mechatronics (AIM). 1999. Atlanta, USA.

9. Vallery, H., et al. Passive and accurate torque control of series elastic actuators. in Intelligent Robots and Systems, 2007. IROS 2007. IEEE/RSJ International Conference on. 2007.

10.Robinson, D.W., Design and Analysis of Series Elasticity in Closed-loop Actuator Force Control, in Department of Mechanical Engineering. 2000, Massachusetts Institute of Technology: Cambringe.

11.Melnykov, A., et al., Linear Elastic Actuator of a Biped Robot "Rotto", in CLAWAR 2011. 2010: Nagoya, Japan. p. 588-595.

12.Kyoungchul, K., B. Joonbum, and M. Tomizuka, Control of Rotary Series Elastic Actuator for Ideal Force-Mode Actuation in Human\&\#x2013;Robot Interaction Applications. Mechatronics, IEEE/ASME Transactions on, 2009. 14(1): p. 105-118. 